\title{
CORRIGENDUM
}

\section{UV-radiation induces dose-dependent regulation of p53 response and modulates p53-HDM2 interaction in human fibroblasts}

Leena Latonen, Yoichi Taya and Marikki Laiho

Correction to: Oncogene (2001) 20, 6784-6793

In the above paper, the key to Figure $6 \mathrm{E}$ was incorrect. The shaded bars represent HDM2 bound p53/total HDM2, and the white bars represent HDM2 bound $\mathrm{p} 53 /$ total $\mathrm{p} 53$. The authors apologise for this error and regret any inconvenience this may have caused. 